ESAIM: PROCEEDINGS, January 2014, Vol. 44, p. 260-275

SMAI Groupe MAS - Journées MAS 2012 - Session thématique

\title{
ON TWO NUMERICAL PROBLEMS IN APPLIED PROBABILITY : DISCRETIZATION OF STOCHASTIC DIFFERENTIAL EQUATIONS AND OPTIMIZATION OF AN EXPECTATION DEPENDING ON A PARAMETER
}

\author{
Aurélien Alfonsi ${ }^{1}$, Benjamin Jourdain ${ }^{2}$, Sophie Laruelle ${ }^{3}$, Sebastian \\ NikLitscheK-Soto ${ }^{4}$ AND Victor ReUtenaueR ${ }^{5}$
}

\begin{abstract}
In the present paper, we first deal with the discretization of stochastic differential equations. We elaborate on the analysis of the weak error of the Euler scheme by Talay and Tubaro 31. to contruct schemes with quicker weak rate of convergence for SDEs corresponding to an infinitesimal generator with smooth coefficients. We also extend this analysis to the case of a discontinuous drift coefficient. In a second part, we present two applications of stochastic gradient algorithms in finance.
\end{abstract}

\section{INTRODUCTION}

This paper groups the contributions of the speakers of the session dedicated to numerical probability organized during the Journées MAS, which took place in Clermont-Ferrand in august 2012. The most basic probabilistic numerical method consists in approximating an expectation of interest by the empirical mean of a sample of $n$ independent random variables distributed according to some law with the same expectation (crude Monte Carlo method and variance reduction techniques). Under the assumption of integrability of these random variables, almost sure convergence of the empirical mean as $n \rightarrow \infty$ is guaranteed by the strong law of large numbers. Under square integrability, one may construct asymptotic confidence intervals based on the central limit theorem to control the statistical error. Some non-asymptotic confidence intervals based on the concentration of measure may also be derived under more stringent assumptions.

In many applications and especially in finance, the expectation of interest is the one of a function $f\left(X_{T}\right)$ of the solution $\left(X_{t}\right)_{t \in[0, T]}$ to a Stochastic Differential Equation, where $T>0$ is a time-horizon. Apart in very restrictive situations, it is not possible to simulate according to the law of $X_{T}$ and one has to resort to timediscretizations of the SDE to construct approximations of this random variable. This introduces a discretization bias called the weak error in the computation of $\mathbb{E}\left[f\left(X_{T}\right)\right]$. For an SDE with smooth coefficients and a smooth function $f$, the weak error of the explicit Euler scheme (the simplest scheme that one can imagine see (2)

\footnotetext{
${ }^{1}$ Université Paris-Est, CERMICS, project team MathRisk ENPC-INRIA-UMLV, Ecole des Ponts, 6-8 avenue Blaise Pascal, 77455 Marne-la-vallée, France. e-mail: alfonsi@cermics.enpc.fr

${ }^{2}$ Université Paris-Est, CERMICS, project team MathRisk ENPC-INRIA-UMLV, Ecole des Ponts, 6-8 avenue Blaise Pascal, 77455 Marne-la-vallée, France. e-mail: jourdain@cermics.enpc.fr

${ }^{3}$ Laboratoire de Mathématiques Appliquées aux Systèmes, École Centrale Paris, Grande Voie des Vignes, F-92 295 ChâtenayMalabry Cedex, France. e-mail: sophie.laruelle@ecp.fr

4 TOSCA project-team, INRIA Sohpia Antipolis - Mediterrane, France, Email: sebastian.niklitschek-soto@inria.fr.

${ }^{5}$ Credit Quantitative Analysis, Citigroup Global Market Limited, Citigroup Center, 33 Canada Square, Canary Wharf, London, United Kingdom. e-mail: victor.reutenauer@citi.com

(C) EDP Sciences, SMAI 2013
} 
has been analyzed theoretically by Talay and Tubaro [31. The first part of the present paper elaborates on the approach introduced by these authors and which relies on the Feynman-Kac parabolic partial differential equation $\partial_{t} u(t, x)=L u(t, x)$ where $L$ is the infinitesimal generator of the diffusion process solving the SDE. When the coefficients of $L$ are smooth (and in particular for affine diffusion processes), we first explain how to construct schemes with higher weak error order than the Euler scheme by decomposing $L$ in an appropriate way. This recent approach addresses the practical need to minimize the weak error for a given computation cost. We also deal with the case of a SDE with a discontinuous drift coefficient. This discontinuity makes the analysis of the weak error introduced by the Euler scheme more delicate than for smooth coefficients. And it deteriorates its rate of convergence to 0 .

The second part of the paper deals with two applications of the Robbins-Monro algorithm to finance. This algorithm is aimed at finding a zero of a function $x \in \mathbb{R}^{d} \mapsto f(x)=\mathbb{E}[F(x, U)]$ where $F: \mathbb{R}^{d} \times \mathbb{R}^{k} \rightarrow \mathbb{R}^{d}$ and $U$ is a $k$-dimensional random vector such that $F(x, U)$ is integrable for each $x \in \mathbb{R}^{d}$. Given a starting point $X_{0}$, a deterministic sequence $\left(\gamma_{n}\right)_{n \geq 1}$ of positive step-sizes and a sequence $\left(U_{n}\right)_{n \geq 1}$ of independent copies of $U$, the algorithm consists in computing inductively

$$
\forall n \geq 0, X_{n+1}=X_{n}-\gamma_{n+1} F\left(X_{n}, U_{n+1}\right)
$$

Theorem 0.1. When

- $\sum_{n \geq 1} \gamma_{n}=+\infty$ and $\sum_{n \geq 1} \gamma_{n}^{2}<+\infty$,

- there exists a unique $x_{\star} \in \mathbb{R}^{d}$ such that $f\left(x_{\star}\right)=0$ and for any compact subset $K$ of $\mathbb{R}^{d} \backslash\left\{x_{\star}\right\}$, $\inf _{x \in K}\left\langle f(x), x-x_{\star}\right\rangle>0$,

- $x \mapsto \mathbb{E}\left[\mid F\left(x,\left.U\right|^{2}\right]\right.$ is at most with quadratic growth,

then the sequence $\left(X_{n}\right)_{n \geq 0}$ converges almost surely to $x_{\star}$.

Of course this algorithm may be used to minimize a convex function $x \in \mathbb{R}^{d} \mapsto v(x)=\mathbb{E}[V(x, U)]$ where $V: \mathbb{R}^{d} \times \mathbb{R}^{k} \rightarrow \mathbb{R}$ by choosing $f(x)=\nabla v(x)$ and $F(x, u)=\nabla_{x} V(x, u)$. The first application presented in Section 2.1 is about the optimal submission of passive orders in a limit order book and the almost sure convergence of the stochastic optimization procedure is proved. The second one presented in Section 2.2 is about the hedging of interest rates derivatives in the presence of a bid-ask spread. For this practical problem, it turns out that the algorithm does not converge well when the criterion to optimize is financially sensible and one has to approximate this criterion by a more convex one.

\section{Discretization of Stochastic DifFEREntial EQUATIONS}

In this part, we focus on the discretization on the time-interval $[0, T]$ of the general Stochastic Differential Equation

$$
X_{t}^{x}=x+\int_{0}^{t} b\left(X_{u}^{x}\right) d u+\int_{0}^{t} \sigma\left(X_{u}^{x}\right) d W_{u}, t \geq 0
$$

that is well defined on a domain $\mathbb{D} \subset \mathbb{R}^{d}$. Namely, $W$ is a standard Brownian motion of dimension $d_{W}$, the coefficients $b: \mathbb{D} \rightarrow \mathbb{R}^{d}$ and $\sigma: \mathbb{D} \rightarrow \mathcal{M}_{d \times d_{W}}(\mathbb{R})$ take respectively their values in $\mathbb{R}^{d}$ and in the set of $d \times d_{W}$ real matrices, and we assume that for any $x \in \mathbb{D}$, weak existence and uniqueness hold for this SDE and $\mathbb{P}\left(\forall t \geq 0, X_{t}^{x} \in \mathbb{D}\right)=1$. Also, we assume that $\exists C>0,\|b(x)\|+\|\sigma(x)\| \leq C(1+\|x\|)$, which gives the uniform boundedness on $[0, T]$ of any moments and excludes explosion in finite time.

To calculate expectations that depend on this SDE, one usually has to sample paths in order to use a MonteCarlo algorithm. Unless in very remarkable cases, it is not possible to sample exactly the law of the SDE increments, and one has to use approximations of the SDE that can be sampled. The most famous one is certainly the Euler-Maruyama discretization scheme, which is defined as follows:

$$
\bar{X}_{t_{0}}=x \text { and for } i \in\{0, \ldots, n-1\}, \bar{X}_{t_{i+1}}=\bar{X}_{t_{i}}+b\left(\bar{X}_{t_{i}}\right)\left(t_{i+1}-t_{i}\right)+\sigma\left(\bar{X}_{t_{i}}\right)\left(W_{t_{i+1}}-W_{t_{i}}\right) \text {. }
$$


Here, we will consider for the sake of simplicity a uniform time grid over the time interval $[0, T]$, i.e. $t_{i}=i T / n$, $0 \leq i \leq n$. We know from the work of Talay and Tubaro 31] that we have the weak error expansion

$$
\mathbb{E}\left[f\left(\bar{X}_{t_{n}}\right)\right]=\mathbb{E}\left[f\left(X_{T}\right)\right]+\frac{c_{1}}{n}+\cdots+\frac{c_{k}}{n^{k}}+O\left(\frac{1}{n^{k+1}}\right),
$$

for any $k \in \mathbb{N}^{*}$ when $f$ is a smooth function and the SDE coefficients are $C^{\infty}$ with bounded derivatives. We say that a discretization scheme $\hat{X}$ has a weak error of order $\nu \in \mathbb{N}^{*}$ when

$$
\left|\mathbb{E}\left[f\left(\hat{X}_{t_{n}}\right)\right]-\mathbb{E}\left[f\left(X_{T}\right)\right]\right|=O\left(1 / n^{\nu}\right),
$$

and the Euler scheme has thus a weak error of order one when the SDE coefficients are smooth.

The expansion (3) enables to perform Romberg extrapolation (Kebaier 17]) and Multilevel Monte-Carlo (Giles [12]). This result has been extended to bounded measurable functions $f$ by Bally and Talay [5] under some hypoellipticity and smoothness assumptions on the SDE coefficients, and to tempered distributions by Guyon [13] under ellipticity and smoothness assumptions.

The present part addresses two recent extensions of these results. First, Section 1.1 deals with the construction of schemes with higher weak error order for SDEs corresponding to an infinitesimal generator with smooth coefficients. Section 1.2 is dedicated to a less regular case in dimension $d=d_{W}=1: \sigma$ is constant but $b$ discontinuous. It turns out that the weak error order of the Euler scheme decreases to $1 / 2-\epsilon$ (see Theorem 1.4.

\subsection{High order discretization schemes for the weak error. Application to affine processes in finance}

One may desire to get more accurate schemes for different reasons. First, these results have only be proved for expectations on the marginal laws (i.e. on European payoffs), so that all the methods that increase the rate of convergence such as Romberg extrapolation are only validated in this case. Getting higher order schemes for the weak error will instead give in general also better approximations for pathwise expectations. This is at least observed in many practical cases even though no theoretical result of this kind has been proved yet. In fact, as we will see, the construction of these schemes requires to better approximate the law of the SDE increments, which explains the better approximation of the path distributions observed in practice. Besides, it is in general still possible to use again extrapolation techniques as it has been shown by Fujiwara [11] and Oshima, Teichmann and Velušček 27 for the case of the Ninomiya and Victoir scheme. Second, for SDEs that are defined on a strict subdomain of $\mathbb{R}^{d}$ (i.e. $\mathbb{D} \subsetneq \mathbb{R}^{d}$ ), the Euler scheme is usually not well defined. In fact, as soon as the matrix $\sigma\left(\hat{X}_{t_{i}}\right)$ has rank $d$ for some time $t_{i}, \hat{X}_{t_{i+1}}$ can take any value in $\mathbb{R}^{d}$ and the Euler scheme is not defined at the next time-step. It is then necessary to consider a different discretization scheme.

The scope of this section is to explain how to get second-order schemes by splitting the infinitesimal generator of the SDE. The idea is to write this operator as the sum of infinitesimal generators of "elementary SDEs". Then, by composition, one can construct a second-order scheme for the main SDE from second-order schemes for the elementary SDEs. In practice, this scheme is easily implemented because its construction is recursive. Besides, if all the elementary SDEs and their associated second-order schemes preserve the domain $\mathbb{D}$, this scheme will be automatically well defined on the domain.

\subsubsection{The weak error analysis}

In this paragraph, we present the framework considered in Alfonsi 4 to analyze the weak error. We define

$$
C_{\mathbf{p o l}}^{\infty}(\mathbb{D})=\left\{f \in C^{\infty}(\mathbb{D}, \mathbb{R}), \forall \alpha \in \mathbb{N}^{d}, \exists C_{\alpha}>0, e_{\alpha} \in \mathbb{N}^{*}, \forall x \in \mathbb{D},\left|\partial_{1}^{\alpha_{1}} \ldots \partial_{d}^{\alpha_{d}} f(x)\right| \leq C_{\alpha}\left(1+\|x\|^{e_{\alpha}}\right)\right\},
$$

the set of smooth functions with derivatives having a polynomial growth. We say that $\left(C_{\alpha}, e_{\alpha}\right)_{\alpha \in \mathbb{N}^{d}}$ is a good sequence for $f$ if $\left|\partial_{1}^{\alpha_{1}} \ldots \partial_{d}^{\alpha_{d}} f(x)\right| \leq C_{\alpha}\left(1+\|x\|^{e_{\alpha}}\right)$ holds for any $x \in \mathbb{D}, \alpha \in \mathbb{N}^{d}$. We assume that the SDE 
coefficients are such that $b \in C_{\mathbf{p o l}}^{\infty}(\mathbb{D})$ and $\sigma \sigma^{*} \in C_{\mathbf{p o l}}^{\infty}(\mathbb{D})$. For affine diffusions, $b(x)$ and $\sigma(x) \sigma^{*}(x)$ are affine functions of $x$, and this condition is clearly satisfied. Then, the infinitesimal generator of (1)

$$
L f(x)=\sum_{i=1}^{d} b_{i}(x) \partial_{i} f(x)+\frac{1}{2} \sum_{i=1}^{d} \sum_{j=1}^{d} \sum_{k=1}^{d_{W}} \sigma_{i, k}(x) \sigma_{j, k}(x) \partial_{i} \partial_{j} f(x)
$$

preserves $C_{\text {pol }}^{\infty}(\mathbb{D})$, i.e. $f \in C_{\text {pol }}^{\infty}(\mathbb{D}) \Longrightarrow L f \in C_{\text {pol }}^{\infty}(\mathbb{D})$.

We would like now to give a framework for the different discretization schemes. When we want to discretize a SDE or more generally a Markov process, it is natural to approximate the law of its increments by a law depending on the starting point $x \in \mathbb{D}$ and the time step $t>0$. We denote by $\left(\hat{p}_{x}(t)(d z), x \in \mathbb{D}, t>0\right)$ such a family of probability laws and by $\hat{X}_{t}^{x}$ a random variable which is sampled according to the distribution $\hat{p}_{x}(t)(d z)$. In the case of the Euler scheme, $\hat{p}_{x}(t)(d z)$ is the probability law of a Gaussian vector with mean $x+t b(x)$ and covariance matrix $t \sigma(x) \sigma^{*}(x)$, and $\hat{X}_{t}^{x}=x+t b(x)+\sigma(x) W_{t}$. Then, we can sample an entire path $\left(\hat{X}_{t_{i}}, 0 \leq i \leq n\right)$ as follows: $\hat{X}_{t_{0}}=x$ and $\hat{X}_{t_{i+1}}$ is sampled conditionally on $\left(\hat{X}_{t_{l}}, 0 \leq l \leq i\right)$ according to the probability measure $\hat{p}_{\hat{X}_{t_{i}}}(T / n)$.

To study the weak error, we will focus on the asymptotic behaviour of

$$
R f(t, x)=\mathbb{E}\left[f\left(\hat{X}_{t}^{x}\right)\right]-\mathbb{E}\left[f\left(X_{t}^{x}\right)\right]
$$

when $t \rightarrow 0^{+}$. We will say that the scheme $\hat{X}_{t}^{x}$ is a potential weak $\nu$-th order scheme if there are constants $C, E, \eta>0$ depending only on a good sequence of $f$ such that

$$
\forall t \in(0, \eta), \forall x \in \mathbb{D},|R f(t, x)| \leq C t^{\nu+1}\left(1+\|x\|^{E}\right)
$$

This means that these constants can be chosen the same for any function $g \in C_{\text {pol }}^{\infty}(\mathbb{D})$ sharing the same good sequence as $f$. By iterating Itô's formula it is easy to check that $\hat{X}_{t}^{x}$ is a potential weak $\nu$-th order scheme if, and only if, the same property is satisfied for $\tilde{R} f(t, x)=\mathbb{E}\left[f\left(\hat{X}_{t}^{x}\right)\right]-\sum_{k=0}^{\nu} \frac{t^{k}}{k !} L^{k} f(x)$. For convenience, we will use the shorthand notation

$$
\mathbb{E}\left[f\left(\hat{X}_{t}^{x}\right)\right]=\sum_{k=0}^{\nu} \frac{t^{k}}{k !} L^{k} f(x)+\operatorname{Rem}\left(t^{\nu+1}\right)
$$

when this property holds true. Obviously, the exact scheme $\hat{X}_{t}^{x}=X_{t}^{x}$ is a potential weak $\nu$-th order scheme for any order $\nu$.

Theorem 1.1. Under the above setting, we assume that

(1) $f: \mathbb{D} \rightarrow \mathbb{R}$ is a function such that $u(t, x)=\mathbb{E}\left[f\left(X_{t}^{x}\right)\right]$ is defined on $[0, T] \times \mathbb{D}, \mathcal{C}^{\infty}$, solves $\forall t \in[0, T], \forall x \in$ $\mathbb{D}, \partial_{t} u(t, x)=L u(t, x)$, and satisfies:

$$
\forall l \in \mathbb{N}, \alpha \in \mathbb{N}^{d}, \exists C_{l, \alpha}, e_{l, \alpha}>0, \forall x \in \mathbb{D}, t \in[0, T],\left|\partial_{t}^{l} \partial_{1}^{\alpha_{1}} \ldots \partial_{d}^{\alpha_{d}} u(t, x)\right| \leq C_{l, \alpha}\left(1+\|x\|^{e_{l, \alpha}}\right)
$$

(2) the scheme $\hat{p}_{x}(t)$ is a potential weak $\nu$ th-order discretization scheme for the operator $L$ and the associated discretization $\left(\hat{X}_{t_{i}}, 0 \leq i \leq n\right)$ has uniformly bounded moments, i.e.

$$
\exists n_{0} \in \mathbb{N}^{*}, \forall q \in \mathbb{N}^{*}, \exists C_{q}>0, \forall n \geq n_{0}, \sup _{0 \leq i \leq n} \mathbb{E}\left[\left\|\hat{X}_{t_{i}}\right\|^{q}\right]<C_{q}
$$

Then, there is $K>0, n_{0} \in \mathbb{N}$, such that $\left|\mathbb{E}\left[f\left(\hat{X}_{t_{n}}\right)\right]-\mathbb{E}\left[f\left(X_{T}^{x}\right)\right]\right| \leq K / n^{\nu}$ for $n \geq n_{0}$.

The proof of this result is given in Alfonsi [4] and mainly relies on the argument used by Talay and Tubaro to analyze the weak error of the Euler scheme. Let us comment briefly the assumptions. The first one only depends 
on the SDE itself: it is true or not, but it does not depend on the scheme chosen. Talay and Tubaro [31] have shown that this holds when the SDE coefficients are $C^{\infty}$ with bounded derivatives and $f$ is $C^{\infty}$ with polynomial growth together with its derivatives. The second assumption brings on the scheme. The uniform bounds on the moments are in general true because this property holds for the SDE thanks to the sublinear growth assumption on the coefficients, and the discretization scheme is meant to approximate this SDE. This property can usually be checked by simple but sometimes tedious calculations. Thus, the main assumption that is required to get a weak error of order $\nu$ is to have a potential weak $\nu$-th order scheme. This is why we focus on this property in the following.

\subsubsection{Scheme composition and operator splitting}

Let us assume that we can write the infinitesimal generator as $L=L_{1}+L_{2}$, where $L_{i}$ is the infinitesimal generator of the SDE $d X_{t}=b_{i}\left(X_{t}\right) d t+\sigma_{i}\left(X_{t}\right) d W_{t}$. We assume that these two SDEs satisfy the same assumptions as (1), and we suppose that $\hat{X}_{t}^{1, x}$ and $\hat{X}_{t}^{2, x}$ are two corresponding potential weak $\nu$-th order schemes that take values in the domain $\mathbb{D}$. For $\lambda_{1}, \lambda_{2}>0$, we consider the random variable $\hat{X}_{\lambda_{2} t}^{2, \hat{X}_{\lambda_{1} t}^{1, x}}$ which consists in first using the scheme for $L_{1}$ with a time step $\lambda_{1} t$ and then the scheme for $L_{2}$ with a time step $\lambda_{2} t$. We denote by

$$
\hat{p}^{2}\left(\lambda_{2} t\right) \circ \hat{p}_{x}^{1}\left(\lambda_{1} t\right)(d z)=\int_{\mathbb{D}} \hat{p}_{y}^{1}\left(\lambda_{2} t\right)(d z) \hat{p}_{x}^{1}\left(\lambda_{1} t\right)(d y)
$$

the corresponding probability measure. Let $f \in C_{\mathbf{p o l}}^{\infty}(\mathbb{D})$. The tower property of the conditional expectation gives $\mathbb{E}\left[f\left(\hat{X}_{\lambda_{2} t}^{2, \hat{X}_{\lambda_{1} t}^{1, x}}\right)\right]=\mathbb{E}\left[\mathbb{E}\left[f\left(\hat{X}_{\lambda_{2} t}^{2, \hat{X}_{\lambda_{1} t}^{1, x}}\right) \mid \hat{X}_{\lambda_{1} t}^{1, x}\right]\right]$, which leads easily to:

$$
\mathbb{E}\left[f\left(\hat{X}_{\lambda_{2} t}^{2, \hat{X}_{\lambda^{1} t}^{1, x}}\right)\right]=\sum_{l_{1}+l_{2} \leq \nu} \frac{\lambda_{1}^{l_{1}} \lambda_{2}^{l_{2}}}{l_{1} ! l_{2} !} t^{l_{1}+l_{2}} L_{1}^{l_{1}} L_{2}^{l_{2}} f(x)+\operatorname{Rem}\left(t^{\nu+1}\right) .
$$

Thus, a potential $\nu$-th order scheme for $L_{i}$ with time step $\lambda_{i} t$ can be seen as the operator $I+\lambda_{i} t L_{i}+\cdots+\frac{\left(\lambda_{i} t\right)^{\nu}}{\nu !} L_{i}^{\nu}$ up to a remainder with order $\nu+1$, and the scheme composition corresponds to the composition of these operators in the reverse order. In the particular case where $L_{1} L_{2}=L_{2} L_{1}$, we get for $\lambda_{1}=\lambda_{2}=1$ :

$$
\mathbb{E}\left[f\left(\hat{X}_{t}^{2, \hat{X}_{t}^{1, x}}\right)\right]=\sum_{k=0}^{\nu} \frac{1}{k !} t^{k}\left(L_{1}+L_{2}\right)^{k} f(x)+\operatorname{Rem}\left(t^{\nu+1}\right),
$$

and $\hat{X}_{t}^{2, \hat{X}_{t}^{1, x}}$ is a potential $\nu$-th order scheme for the SDE associated to $L_{1}+L_{2}$. In general, $L_{1}$ and $L_{2}$ do not commute. It is however still possible to get second-order schemes for $L_{1}+L_{2}$ from second order schemes for $L_{1}$ and $L_{2}$. Let us denote $S_{i}(t)=I+t L_{i}+\frac{t^{2}}{2} L_{i}^{2}+\ldots$ the formal series where the dots indicate terms of order equal or higher than 3 in $t$. Then, we get by simple calculations the following identities:

$$
\begin{aligned}
S_{1}(t / 2) S_{2}(t) S_{1}(t / 2) & =I+t\left(L_{1}+L_{2}\right)+\frac{t^{2}}{2}\left(L_{1}+L_{2}\right)^{2}+\ldots \\
\frac{1}{2}\left(S_{1}(t) S_{2}(t)+S_{2}(t) S_{1}(t)\right) & =I+t\left(L_{1}+L_{2}\right)+\frac{t^{2}}{2}\left(L_{1}+L_{2}\right)^{2}+\ldots
\end{aligned}
$$

Thus, if $B$ denote an independent Bernoulli variable of parameter $1 / 2$, we get that $\hat{X}_{t / 2}^{1, \hat{X}_{t}^{2, \hat{X}_{t / 2}^{1, x}}}$ (also known as Strang splitting 29. in the field of ODEs) and $B \hat{X}_{t}^{2, \hat{X}_{t}^{1, x}}+(1-B) \hat{X}_{t}^{1, \hat{X}_{t}^{2, x}}$ are potential second order schemes for $L_{1}+L_{2}$. Their corresponding probability laws are $\hat{p}^{1}(t / 2) \circ \hat{p}^{2}(t) \circ \hat{p}_{x}^{1}(t / 2)$ and $\frac{1}{2}\left(\hat{p}^{2}(t) \circ \hat{p}_{x}^{1}(t)+\hat{p}^{1}(t) \circ \hat{p}_{x}^{2}(t)\right)$. More generally, let us assume that for any $i \in\{1, \ldots, m\}, \hat{p}_{x}^{i}(t)$ is a potential second order scheme for $L_{i}$ that 
takes values in $\mathbb{D}$. Then, we get by the same argument that $\frac{1}{2}\left(\hat{p}^{m}(t) \circ \cdots \circ \hat{p}_{x}^{1}(t)+\hat{p}^{1}(t) \circ \cdots \circ \hat{p}_{x}^{m}(t)\right)$ is a potential second order scheme for $L_{1}+\cdots+L_{m}$.

\subsubsection{The Ninomiya and Victoir scheme}

We have seen in the last paragraph how to get a second order scheme by splitting its infinitesimal generator in operators for which we know second order schemes. A natural question is then how to split it. Of course, there are many different ways to write an infinitesimal generator as the sum of infinitesimal generators. Among them, the splitting considered by Ninomiya and Victoir [26] is remarkable since it reduces the problem of approximating SDEs to the problem of approximating ODEs. Let us explain how it works.

The Ninomiya and Victoir splitting is given by $L=V_{0}+\frac{1}{2} \sum_{k=1}^{d_{W}} V_{k}^{2}$, where:

$$
\begin{aligned}
& V_{0} f(x)=\sum_{i=1}^{d} b_{i}(x) \partial_{i} f(x)-\frac{1}{2} \sum_{i, j=1}^{d} \sum_{k=1}^{d_{W}} \partial_{j} \sigma_{i, k} \sigma_{j, k} \partial_{i} f(x) \\
& V_{k} f(x)=\sum_{i=1}^{d} \sigma_{i, k}(x) \partial_{i} f \text { for } k=1, \ldots, d_{W} .
\end{aligned}
$$

By simple calculations, we can check that, for $1 \leq k \leq d_{W}, \frac{1}{2} V_{k}^{2}$ is the infinitesimal generator of the SDE $d X_{t}^{k}=\sigma\left(X_{t}^{k}\right) \circ d W_{t}^{\{k\}}$, where $\left(W_{t}^{\{k\}}\right)_{i}=\mathbf{1}_{i=k}\left(W_{t}\right)_{i}$ for $1 \leq i \leq d_{W}$ and $\circ$ denotes the Stratonovitch integral. Denoting by $v_{0}(x)$ the sum of $b(x)$ and of the vector $\left(-\frac{1}{2} \sum_{j=1}^{d} \sum_{k=1}^{d_{W}} \partial_{j} \sigma_{i, k} \sigma_{j, k}(x)\right)$, and by $v_{k}(x)$ the $k$-th column of the matrix $\sigma(x)$ for $1 \leq k \leq d_{W}$, we have $V_{k} f(x)=v_{k}(x)$. $\nabla f$ for $k=0, \ldots, d_{W}$. We assume that $\exists K>0,\left\|v_{k}(x)\right\| \leq K(1+\|x\|)$ and that $X_{0}(t, x)$ (resp. $\left.X_{k}(t, x), k=1, \ldots, d_{W}\right)$ is a $\mathbb{D}$-valued solution to the ODE

$$
\frac{d X_{0}(t, x)}{d t}=v_{0}\left(X_{0}(t, x)\right), t \geq 0 \quad\left(\operatorname{resp} . \frac{d X_{k}(t, x)}{d t}=v_{k}\left(X_{k}(t, x)\right), t \in \mathbb{R}\right)
$$

that starts from $x \in \mathbb{D}$ at $t=0$. Then, we can easily check that $d X_{k}\left(\left(W_{t}\right)_{k}, x\right)=\sigma\left(X_{k}\left(\left(W_{t}\right)_{k}, x\right)\right) \circ d W_{t}^{\{k\}}$ which means that $X_{k}\left(\left(W_{t}\right)_{k}, x\right)$ is an exact scheme for SDE associated to $\frac{1}{2} V_{k}^{2}$. Thus, if we are able to solve explicitly these ODEs, we can get by composition a potential second order scheme for the main SDE. More precisely, let $\hat{p}_{x}^{k}(t)(d z)$ denote the probability measure of $X_{k}\left(\left(W_{t}\right)_{k}, x\right)$ and $\hat{p}_{x}^{0}(t)(d z)=\delta_{X_{0}(t, x)}(d z)$ be the Dirac mass at $X_{0}(t, x)$. Then, Ninomiya and Victoir [26] propose the following potential second order scheme:

$$
\frac{1}{2}\left(\hat{p}^{0}(t / 2) \circ \hat{p}^{d_{W}}(t) \circ \cdots \circ \hat{p}^{1}(t) \circ \hat{p}_{x}^{0}(t / 2)+\hat{p}^{0}(t / 2) \circ \hat{p}^{1}(t) \circ \cdots \circ \hat{p}_{x}^{d_{W}}(t) \circ \hat{p}_{x}^{0}(t / 2)\right) .
$$

In general, there is no available explicit solution for $X_{0}$ and $X_{k}$, but Ninomiya and Ninomiya [25] show that $\hat{X}_{0}(t, x)$ and $\hat{X}_{k}\left(\left(W_{t}\right)_{k}, x\right)$ are potential second order schemes for $V_{0}$ and $\frac{1}{2} V_{k}^{2}$ provided that $\hat{X}_{0}$ and $\hat{X}_{k}$ are respectively third and sixth order approximations (i.e. $f\left(\hat{X}_{0}(t, x)\right)-f\left(X_{0}(t, x)\right)=\operatorname{Rem}\left(t^{3}\right)$ and $f\left(\hat{X}_{k}(t, x)\right)-$ $f\left(X_{k}(t, x)\right)=\operatorname{Rem}\left(t^{6}\right)$ for $\left.f \in C_{\text {pol }}^{\infty}(\mathbb{D})\right)$. Such approximations can be obtained by using Runge-Kutta methods. Last, we also preserve the second-order convergence if we replace the Brownian increments by $\sqrt{t} Y$, where $Y$ is a bounded random variable that matches the five first moments of the standard Gaussian variable.

\subsubsection{The case of the CIR process, and beyond}

In this paragraph, we focus on applying the previous results to the Cox-Ingersoll-Ross (CIR) process:

$$
\left\{\begin{array}{l}
X_{t}^{x}=x+\int_{0}^{t}\left(a-k X_{s}^{x}\right) d s+\sigma \int_{0}^{t} \sqrt{X_{s}^{x}} d W_{s}, t \in[0, T] \\
x \geq 0
\end{array}\right.
$$

with parameters $(a, k, \sigma) \in \mathbb{R}_{+}^{*} \times \mathbb{R} \times \mathbb{R}_{+}$. This affine process is also known as the squared Bessel process when $k=0$. It is nonnegative and its domain is thus $\mathbb{D}=\mathbb{R}_{+}$. 
First, let us mention that the Euler scheme is not well defined for this SDE. In fact, the Brownian increment can always lead the scheme to negative values, and it is then not possible to calculate the scheme at the next time step since the square-root is not defined. Therefore, one has to consider appropriate schemes for this SDE. Many different schemes have been proposed in the literature (see references in [4]), however most of them fail to be accurate when the volatility is high $\left(\sigma^{2}>4 a\right.$ to be precise). Here, we present the second order scheme given in [4] which works without restriction on the parameters. As we will see, this scheme is an adaptation of the Ninomiya and Victoir scheme.

Let us then first write the Ninomiya and Victoir scheme for the CIR. The infinitesimal generator of the CIR is

$$
L f(x)=(a-k x) \partial_{x} f(x)+\frac{1}{2} \sigma^{2} x \partial_{x}^{2} f(x)=V_{0} f(x)+\frac{1}{2} V_{1}^{2} f(x),
$$

with $V_{0} f(x)=\left(a-k x-\frac{\sigma^{2}}{4}\right) f^{\prime}(x)$ and $V_{1} f(x)=\sigma \sqrt{x} f^{\prime}(x)$. Each part can be solved explicitly. Let $\psi_{k}(t)=\frac{1-e^{-k t}}{k}$ when $k \neq 0$ and $\psi_{0}(t)=t$ otherwise. Then, $X_{0}(t, x)=x e^{-k t}+\left(a-\sigma^{2} / 4\right) \psi_{k}(t)$ solves $\frac{d}{d t} X_{0}(t, x)=a-k X_{0}(t, x)-$ $\frac{\sigma^{2}}{4}$ and the infinitesimal generator of the process $X_{1}\left(W_{t}, x\right)=\left(\sqrt{x}+\frac{\sigma}{2} W_{t}\right)^{2}$ is $\frac{1}{2} V_{1}^{2}$. Using the Strang splitting $X_{0}\left(t / 2, X_{1}\left(W_{t}, X_{0}(t / 2, x)\right)\right)$, we then get the Ninomiya and Victoir scheme for the CIR (see [26]):

$$
\hat{X}_{t}^{x}=e^{-\frac{k t}{2}}\left(\sqrt{\left(a-\sigma^{2} / 4\right) \psi_{k}(t / 2)+e^{-\frac{k t}{2}} x}+\frac{\sigma}{2} W_{t}\right)^{2}+\left(a-\sigma^{2} / 4\right) \psi_{k}(t / 2) .
$$

Unfortunately, this scheme is only well defined when $\sigma^{2} \leq 4 a$. In this case, we can check easily that $X_{0}(t, x) \in \mathbb{R}_{+}$ for any $x \in \mathbb{R}_{+}$, and the composition is well defined. In contrast, this is no longer true when $\sigma^{2}>4 a$. Let $x \geq 0$. When $x$ is close to zero, $X_{0}(t / 2, x)$ is negative and $X_{1}\left(W_{t}, X_{0}(t / 2, x)\right)$ is not defined. When $x$ is larger, $X_{1}\left(W_{t}, X_{0}(t / 2, x)\right)$ is well defined but can be close to zero with some positive probability due to the Gaussian law. Thus, we still have $\mathbb{P}\left(\hat{X}_{t}^{x}<0\right)>0$ in this case. Once the scheme is fallen into negative values, it is not well defined at the next time-step.

To correct this, the idea is to replace the Gaussian increment by a bounded random variable that matches the five first moments of the normal distribution. As we have seen, this preserves the second-order convergence rate. Here is a possible choice:

$$
\mathbb{P}(Y=\sqrt{3})=\frac{1}{6}, \mathbb{P}(Y=-\sqrt{3})=\frac{1}{6}, \text { and } \mathbb{P}(Y=0)=\frac{2}{3}
$$

With this choice, we can easily checked that the following scheme

$$
\hat{X}_{t}^{x}=e^{-\frac{k t}{2}}\left(\sqrt{\left(a-\sigma^{2} / 4\right) \psi_{k}(t / 2)+e^{-\frac{k t}{2}} x}+\frac{\sigma}{2} \sqrt{t} Y\right)^{2}+\left(a-\sigma^{2} / 4\right) \psi_{k}(t / 2)
$$

is well defined when $x \geq \mathbf{1}_{\left\{\sigma^{2}>4 a\right\}} e^{\frac{k t}{2}}\left(\left(\frac{\sigma^{2}}{4}-a\right) \psi_{k}(t / 2)+\left[\sqrt{e^{\frac{k t}{2}}\left[\left(\frac{\sigma^{2}}{4}-a\right) \psi_{k}(t / 2)\right]}+\frac{\sigma}{2} \sqrt{3 t}\right]^{2}\right)=: \mathbf{K}_{2}(t)$.

When $x \in\left[0, \mathbf{K}_{2}(t)\right)$, one has to consider a different scheme. Roughly speaking, it is sufficient to find a scheme $\hat{X}_{t}^{x}$ that matches the two first moments of $X_{t}^{x}$. In fact, we have to check that $\mathbb{E}\left(f\left(\hat{X}_{t}^{x}\right)\right)-\mathbb{E}\left(f\left(X_{t}^{x}\right)\right)=R e m\left(t^{3}\right)$ for $f \in C_{\mathbf{p o l}}^{\infty}(\mathbb{D})$. The Taylor expansion of order 3 around 0 gives $f(x)=f(0)+f^{\prime}(0) x+\frac{f^{\prime \prime}(0)}{2} x^{2}+\int_{0}^{x} \frac{(x-y)^{2}}{2} f^{(3)}(y) d y$. Since $\hat{X}_{t}^{x}$ matches the two first moments of $X_{t}^{x}$ and $\left|f^{(3)}(y)\right| \leq C\left(1+|y|^{q}\right)$, we get $\left|\mathbb{E}\left(f\left(\hat{X}_{t}^{x}\right)\right)-\mathbb{E}\left(f\left(X_{t}^{x}\right)\right)\right| \leq$ $C \mathbb{E}\left[\left(\hat{X}_{t}^{x}\right)^{3}+\left(\hat{X}_{t}^{x}\right)^{q+3}+\left(X_{t}^{x}\right)^{3}+\left(X_{t}^{x}\right)^{q+3}\right]$. Since $x \leq \mathbf{K}_{2}(t)=O(t)$, this can be upper bounded by $C t^{3}$ provided that the scheme $\hat{X}_{t}^{x}$ is suitably chosen. A possible choice is

$$
\hat{X}_{t}^{x}=\mathbf{1}_{\{U \leq \pi(t, x)\}} \frac{\mathbb{E}\left[X_{t}^{x}\right]}{2 \pi(t, x)}+\mathbf{1}_{\{U>\pi(t, x)\}} \frac{\mathbb{E}\left[X_{t}^{x}\right]}{2(1-\pi(t, x))} \text { with } \pi(t, x)=\frac{1-\sqrt{1-\mathbb{E}\left[X_{t}^{x}\right]^{2} / \mathbb{E}\left[\left(X_{t}^{x}\right)^{2}\right]}}{2}
$$


where $U$ is a uniform random variable on $[0,1]$. Let us recall that $\mathbb{E}\left[X_{t}^{x}\right]=x e^{-k t}+a \psi_{k}(t)$ and $\mathbb{E}\left[\left(X_{t}^{x}\right)^{2}\right]=$ $\mathbb{E}\left[X_{t}^{x}\right]^{2}+\sigma^{2} \psi_{k}(t)\left[a \psi_{k}(t) / 2+x e^{-k t}\right]$, so that everything can be calculated explicitly.

We can now apply Theorem 1.1. We know by $\left[3\right.$ that $u(t, x)=\mathbb{E}\left[f\left(X_{T-t}^{x}\right)\right]$ satisfies the required assumption and by [4] that the scheme has uniformly bounded moments.

Theorem 1.2. Let $\hat{X}_{t}^{x}$ denote the scheme (8) for $x \geq \mathbf{K}_{2}(t)$ and the scheme $(9)$ for $x \in\left[0, \mathbf{K}_{2}(t)\right)$. Let $\left(\hat{X}_{t_{i}}, 0 \leq i \leq n\right)$ denote the associate discretization on the regular time grid starting from $\hat{X}_{t_{0}}=x$. We have:

$$
\forall f \in C_{\text {pol }}^{\infty}\left(\mathbb{R}_{+}\right), \exists K>0, \forall n \in \mathbb{N}^{*},\left|\mathbb{E}\left[f\left(\hat{X}_{t_{n}}\right)\right]-\mathbb{E}\left[f\left(X_{T}^{x}\right)\right]\right| \leq K / n^{2}
$$

Using similar ideas, it is also possible in the case of the CIR diffusion to construct a third order scheme. Numerical tests comparing these two schemes to other numerical schemes are given in [4]. Then, still by using operator splitting and scheme composition, we can easily construct second order schemes for the Heston model [14] and for the Affine Term Structure Model proposed by Dai and Singleton [9] for interest rate modelling. Details are given in 4]. These schemes have been implemented in the free Premia software to calculate Put and Call option prices in the Heston model and can be run online at https://quanto.inria.fr. Still by using the same techniques, we can also construct second-order schemes for Wishart processes and affine SDEs on semidefinite positive matrices. The Cox-Ingersoll-Ross process can in fact be seen as a particular case of these processes in dimension 1. To get such schemes, we exhibit in [2] a remarkable splitting of the infinitesimal generator of Wishart processes as the sum of commuting operators that are associated to elementary Wishart processes. These elementary processes can be exactly sampled with one CIR increment (a non centered $\chi$ square distribution) and Gaussian variables. Replacing these variables by the second-order scheme for the CIR and by the moment-matching variable $Y$ given by (7), we get a second-order scheme for these elementary processes. Then, by composition, we get a second-order scheme for any Wishart processes or affine diffusions on semidefinite positive matrices. Also from this remarkable splitting, it is possible to construct autonomous SDEs that are well defined on the set of correlation matrices. These correlation processes naturally extend the well known Wright-Fisher process and have many remarkable properties such as explicit calculations of their moments. We can also easily construct second-order schemes for these processes (see [1] for the details).

\subsection{Discretization of one-dimensional stochastic differential equations with discontinous drift}

Let $b$ be a function in the set $C_{b}^{3}(\mathbb{R}-\{0\})$, where, for $k \in \mathbb{N}, C_{b}^{k}(\mathbb{R}-\{0\})$ denotes the set of functions bounded together with their derivatives up to the order $k$ on $\mathbb{R}-\{0\}$. We assume also that the derivative of the function $b$ has finite limits at both left and right sides of the origin. Consider the following parabolic problem,

$$
\left\{\begin{array}{ccc}
\partial_{t} u(t, x) & =L u(t, x), & (t, x) \in] 0, T] \times \mathbb{R}-\{0\} \\
u(0, x) & =f(x), & x \in \mathbb{R} \\
u(t, 0+) & =u(t, 0-), & t \in] 0, T[ \\
\partial_{x} u(t, 0+) & =\partial_{x} u(t, 0-), &
\end{array},\right.
$$

where $L=\frac{1}{2} \partial_{x x} u(t, x)+b(x) \partial_{x} u(t, x)$ and the transmission conditions are compatibility conditions imposed to the function $u$ or to its derivatives at the interface. In the sequel we will refer to this problem as the Transmission Problem. The existence and uniqueness of a continuous solution for this problem has been shown for example in [20] (see Theorem 13.2, Chapter 6).

The first aim of this work will be to give a probabilistic interpretation of this problem. This kind of interpretation will allow us to show accurate pointwise estimates on the function $u$ and its derivatives. Then we will show that the stochastic process associated to our probabilistic interpretation can be approximated using the Euler method and that the rate of convergence of this kind of approximation is the same as the one found by Martinez and Talay [23] in a different and complementary framework. The fact that the rates of convergence in both cases are the same, permits to extend the results shown in this article to the framework in which both the drift and the diffusion coefficients have a common and finite number of discontinuities (see [24]). 


\subsubsection{Probabilistic interpretation of the transmission problem}

For each $x \in \mathbb{R}$ we consider a stochastic process $X^{x}$ solution to 11 in dimension $d=d_{W}=1$ and in the particular case of a diffusion coefficient $\sigma$ constant and equal to 1 :

$$
X_{t}^{x}=x+\int_{0}^{t} b\left(X_{s}^{x}\right) d s+W_{t}
$$

Here $\left(W_{t}\right)_{t \geq 0}$ is a standard one-dimensional Brownian motion $\left(d_{W}=1\right)$. The sense of this last equality can be found for exemple in [16] Section 5.3 (see Proposition 3.6 and Corolary 3.11).

The next result gives a probabilistic interpretation to the Transmission Problem (10) using the process $X^{x}$.

Theorem 1.3. Suppose $b \in C_{b}^{3}\left(\mathbb{R}^{+}\{0\}\right)$ and that the values $b^{(i)}(0-)$ and $b^{(i)}(0+)$ are well defined for $i \in\{0,1\}$. Let $f$ be a function such that its derivatives have at most polynomial growth and belonging to the set

$$
\mathcal{W}^{2}=\left\{h \in C^{2}(\mathbb{R}-\{0\}), h^{\prime}(0+)=h^{\prime}(0-)\right\} .
$$

Then the function $u(x, t)=\mathbb{E}\left[f\left(X_{t}^{x}\right)\right],(t, x) \in[0, T] \times \mathbb{R}$ belongs to the set $C_{b}^{1,2}([0, T] \times(\mathbb{R}-\{0\})) \cap$ $C([0, T] \times \mathbb{R})$ and solves the Transmission Problem $[10]$.

\subsubsection{Some estimates for the derivatives of $u$}

In the next theorem we show that the derivatives of the function $u$ have at most polynomial growth.

Theorem 1.4. Suppose that $f$ is a function such that all its derivatives up to the order four have polynomial growth belonging to the set

$$
\mathcal{W}^{4}=\left\{h \in C^{4}(\mathbb{R}-\{0\}), h^{\prime}(0+)=h^{\prime}(0-),(\mathcal{L} h)^{\prime}(0+)=(\mathcal{L} h)^{\prime}(0-)\right\},
$$

then for every $x \neq 0, t \in[0, T], k \in\{0,1,2\}$ and $j \in\{0,1, \ldots, 4\}$ there exists a contant $C>0$ and a positive integer $p$, which depends only on $b, T$, and the function $f$ and its derivatives up to order four, such that

$$
\left|\partial_{t^{k}} u(t, x)\right| \leq C\left(1+|x|^{2 p}\right) \text { and }\left|\partial_{x^{j}} u(t, x)\right| \leq C\left(1+|x|^{2 p}\right) .
$$

\subsubsection{Euler scheme and rate of convergence}

For any positive integer $n$, the Euler scheme (2) associated to 111 writes

$$
\bar{X}_{t_{0}}=x \text { and for } i \in\{0, \ldots, n-1\}, \bar{X}_{t_{i+1}}=\bar{X}_{t_{i}}+b\left(\bar{X}_{t_{i}}\right)\left(t_{i+1}-t_{i}\right)+W_{t_{i+1}}-W_{t_{i}} \text {, }
$$

where $t_{i}=i T / n, 0 \leq i \leq n$.

The next result shows us that scheme allows us to properly approximate the weak solution of (11).

Theorem 1.5. Let $b \in C_{b}^{3}\left(\mathbb{R}^{+}\{0\}\right)$ such that the values $b^{(i)}(0-)$ and $b^{(i)}(0+)$ are well defined for $i \in\{0,1\}$, and $f \in \mathcal{W}^{4}$ a function such that all its derivatives up to the order four have polynomial growth. Then there exists a positive constant $C$ and an integer $p$, such that for all $0<\epsilon<\frac{1}{2}$ and $n$ large enough and $x \in \mathbb{R}$,

$$
\left|\mathbb{E}\left[f\left(X_{T}^{x}\right)\right]-\mathbb{E}\left[f\left(\bar{X}_{T}\right)\right]\right| \leq C\left(1+|x|^{2 p}\right)\left(\frac{T}{n}\right)^{\frac{1}{2}-\epsilon}
$$

\section{Applichtions OF StOChastic ALGORITHMS IN FINANCE}

We present two different problems using stochastic gradient algorithms to optimize strategies. The first work is about the optimal submission of passive orders in a limit order book, the second one is about the 
hedging of interest rates derivative products in the presence of a bid-ask spread. In the first part, we model a trader interacting with a continuous market as an iterative algorithm that adjusts limit prices at a given rhythm and propose a procedure to minimize trading costs. We prove the a.s. convergence of the algorithm under assumptions on the cost function and give some practical criteria on model parameters to ensure that the conditions to use the algorithm are met (notably, using the co-monotony principle). In the second part, we study in the presence of bid-ask spread costs the optimal strategy of hedging of an interest rates derivative swap that pays a fixed rate against a floating rate.

\subsection{Optimal posting price of limit orders using stochastic optimization}

We study here the optimal distance to submit passive orders in a limit order book, without needing a model of the limit order book dynamics (see 21] for more details). We consider an agent who wants to buy during a short period $[0, T]$ a quantity $Q_{T}$ of traded assets and we look for the optimal distance where he has to post his limit order to minimize the execution cost (the case of a sell limit order may be obtained by symmetry). Our approach follows the mechanism of a "learning trader": he will guess the optimal posting distance (the price that balances adverse selection and non execution risk), by successive trials, errors and corrections. The optimal recursive procedure derived from our framework gives the best price adjustment to apply to an order for a given stopping time (reassessment dates) given past market observations.

We model the execution flow of orders at time $T$ by a random variable $N_{T}^{(\delta)}$ whose conditional distribution with respect to the price process over $[0, T]$ is Poisson with parameter depending on the fair price and the distance of order submission $\delta$. The execution cost results from the sum of the price of the executed quantity and a penalty function depending on the remaining quantity to be executed at the end of the period $[0, T]$. This penalty models the excess cost induced by crossing the spread and the resulting market impact of this execution. The aim is to find the optimal distance $\delta^{*}$ that minimizes the execution cost. This leads to an optimization problem under constraints which we solve by using a recursive stochastic procedure with projection (see 18 . and $[19]$. We prove the a.s. convergence of the constrained algorithm under additional assumptions on the execution cost function. From a practical point of view, it is not easy to check the conditions on the cost function, so we give criteria on the model parameters that ensure the viability of the algorithm by relying on the co-monotony principle we assume the "fair price" process $\left(S_{t}\right)_{t \in[0, T]}$ satisfies.

The Section 2.1 is organized as follows: in Section 2.1.1 we first propose a model for the execution process of posted orders, then we define a penalized execution cost function and devise the stochastic recursive procedure under constraints. Section 2.1.2 states the main convergence results and Section 2.1.3 provide operating criteria that ensure this convergence, based on a co-monotony principle for one dimensional diffusions.

Notations. $\bullet(x)_{+}=\max \{x, 0\}, \llbracket 0, x \rrbracket:=\prod_{i=1}^{d}\left[0, x_{i}\right],\langle\cdot \mid \cdot\rangle$ denotes the canonical inner product on $\mathbb{R}^{d}$.

- $\mathbb{D}([0, T], A):=\{f:[0, T] \rightarrow A$ càdlàg $\}$ (equipped with the Skorokhod topology when necessary see [15]) where càdlàg means right continuous with left limits and $A=\mathbb{R}^{q}, \mathbb{R}_{+}^{q}$, etc. They are equipped with the standard Borel $\sigma$-field $\sigma(\alpha \mapsto \alpha(t), t \in[0, T])$.

- $\|\alpha\|_{\infty}=\sup _{t \in[0, T]}|\alpha(t)|, \alpha \in \mathbb{D}([0, T], \mathbb{R})$ and $f_{\ell}^{\prime}$ denotes the left derivative of $f$.

\subsubsection{Modeling and design of the algorithm}

We focus our work on the problem of optimal trading with limit buy orders on one security without needing to model the limit order book dynamics. We only model the execution flow which reaches the price where the limit order is posted on a short period $T$ with a random variable $N_{T}^{(\delta)}$ whose conditional distribution with respect to $\left(S_{t}\right)_{t \geq 0}$ is Poisson with parameter $\Lambda_{T}(\delta, S):=\int_{0}^{T} \lambda\left(S_{t}-\left(S_{0}-\delta\right)\right) d t$, where $0 \leq \delta \leq \delta_{\max }\left(\delta_{\max } \in\left(0, S_{0}\right)\right.$ is the depth of the order book), $\lambda:\left[-S_{0},+\infty\right) \rightarrow \mathbb{R}_{+}$is a finite non-increasing convex function and $\left(S_{t}\right)_{t \geq 0}$ is a stochastic process modeling the dynamics of the best bid price of a security stock.

Over the period $[0, T]$, we aim to execute a portfolio of size $Q_{T} \in \mathbb{N}$ invested in the asset $S$. The execution cost for a distance $\delta$ is $\mathbb{E}\left[\left(S_{0}-\delta\right)\left(Q_{T} \wedge N_{T}^{(\delta)}\right)\right]$. We add to this execution cost a market impact penalty function $\Phi: \mathbb{R} \mapsto \mathbb{R}_{+}$, non-decreasing and convex, with $\Phi(0)=0$, to model the additional cost of the execution of the 
remaining quantity $\left(Q_{T}-N_{T}^{(\delta)}\right)_{+}$. Then the resulting cost of execution on a period $[0, T]$ reads

$$
C(\delta):=\mathbb{E}\left[\left(S_{0}-\delta\right)\left(Q_{T} \wedge N_{T}^{(\delta)}\right)+\kappa S_{T} \Phi\left(\left(Q_{T}-N_{T}^{(\delta)}\right)_{+}\right)\right]
$$

where $\kappa>0$ is a free tuning parameter. Our aim is then to minimize this cost by choosing the distance to post at. Our strategy to solve $\min _{0 \leq \delta \leq \delta_{\max }} C(\delta)$ numerically is to take advantage of the representation of $C$ and its first two derivatives as expectations to devise a stochastic gradient procedure to find the minimum of the (penalized) cost function. Furthermore we will show that under natural assumptions on $Q_{T}$ and $\kappa, C$ is twice differentiable and strictly convex on $\left[0, \delta_{\max }\right]$ with $C^{\prime}(0)<0$. Consequently,

$$
\operatorname{argmin}_{\delta \in\left[0, \delta_{\max }\right]} C(\delta)=\left\{\delta^{*}\right\}, \quad \delta^{*} \in\left(0, \delta_{\max }\right] \quad \text { and } \quad \delta^{*}=\delta_{\max } \quad \text { iff } \quad C \text { is non-increasing on }\left[0, \delta_{\max }\right] .
$$

We specify representations as expectations of $C^{\prime}$ (see [21]) by exhibiting a Borel functional

$$
H:\left[0, \delta_{\max }\right] \times \mathbb{D}([0, T], \mathbb{R}) \longrightarrow \mathbb{R} \quad \text { such that } \quad \forall \delta \in\left[0, \delta_{\max }\right], \quad C^{\prime}(\delta)=\mathbb{E}[H(\delta, S)],
$$

where, by denoting by $N^{\mu}$ a Poisson random variable with parameter $\mu$, we have

$$
\begin{aligned}
H(\delta, S)= & -Q_{T} \mathbb{P}\left(N^{\mu}>Q_{T}\right)_{\mid \mu=\Lambda_{T}(\delta, S)}-\kappa S_{T} \frac{\partial}{\partial \delta} \Lambda_{T}(\delta, S) \varphi^{(\delta)}(\mu) \\
& +\left(\frac{\partial}{\partial \delta} \Lambda_{T}(\delta, S)\left(S_{0}-\delta\right)-\Lambda_{T}(\delta, S)\right) \mathbb{P}\left(N^{\mu} \leq Q_{T}-1\right)_{\mid \mu=\Lambda_{T}(\delta, S)}
\end{aligned}
$$

with $\varphi^{(\delta)}(\mu)=\mathbb{E}\left[\left(\Phi\left(Q_{T}-N^{\mu}\right)-\Phi\left(Q_{T}-N^{\mu}-1\right)\right) \mathbb{1}_{\left\{N^{\mu} \leq Q_{T}-1\right\}}\right]_{\mid \mu=\Lambda_{T}(\delta, S)}$.

In practice, to implement numerically the recursive procedure, we have to replace the "copies" $S^{(n)}$ by copies $\bar{S}^{(n)}$ of a time discretization $\bar{S}=\left(\bar{S}_{t_{i}}\right)_{0 \leq i \leq m}$, typically an Euler scheme of a Brownian diffusion process (see (1) and (2)). Then, with an obvious abuse of notation for the function $H$, we can write the implementable procedure as follows:

$$
\delta_{n+1}=\operatorname{Proj}_{\left[0, \delta_{\max }\right]}\left(\delta_{n}-\gamma_{n+1} H\left(\delta_{n},\left(\bar{S}_{t_{i}}^{(n+1)}\right)_{0 \leq i \leq m}\right)\right), n \geq 0, \delta_{0} \in\left[0, \delta_{\max }\right],
$$

where $\left(\bar{S}_{t_{i}}^{(n)}\right)_{0 \leq i \leq m}$ are copies of $\left(\bar{S}_{t_{i}}\right)_{0 \leq i \leq m}$ either independent or sharing "ergodic" properties, namely some averaging properties in the sense of 22 . In the first case, one will think about simulated data after a calibration process and in the second case to a direct implementation using a historical high frequency database of best opposite prices of the asset $S$.

\subsubsection{Main convergence results}

The following theorems give a.s. convergence results for the stochastic procedure (14): the first one for i.i.d. sequences and the second one for "averaging" sequences (see [22]).

I.i.d. simulated data from a formerly calibrated model. We consider an innovation process $\left\{\left(\bar{S}_{t_{i}}^{(n)}\right)_{0 \leq i \leq m}, n \geq\right.$ $0\}$ coming from a diffusion model beforehand calibrated on real data, which can be simulated at time $t_{i}$, $0 \leq i \leq m$, either exactly or via a stepwise constant time discretization scheme.

Theorem 2.1. Assume the cost function $\bar{C}$ related to the discretization scheme $\left(\bar{S}_{t}\right)_{t \in[0, T]}$ is strictly convex $\left[0, \delta_{\max }\right]$ with $\bar{C}^{\prime}(0)<0$. Furthermore, assume that the decreasing step sequence satisfies the standard "decreasing step assumption"

$$
\sum_{n \geq 1} \gamma_{n}=+\infty \quad \text { and } \quad \sum_{n \geq 1} \gamma_{n}^{2}<+\infty
$$

Let $\left(\bar{S}_{t_{i}}^{(n)}\right)_{0 \leq i \leq m}, n \geq 1$, be a sequence of i.i.d. copies of $\left(\bar{S}_{t_{i}}\right)_{0 \leq i \leq m}$, then the recursive procedure defined by 14) converges a.s. towards its target $\bar{\delta}^{*}=\operatorname{argmin}_{\delta \in\left[0, \delta_{\max }\right]} \bar{C}(\delta)$. 
This theorem is a straightforward application of the classical a.s. convergence for constrained stochastic algorithms (see [18, 19]).

Direct implementation on a historical high frequency dataset sharing averaging properties. We will assume that the price sequence $\left\{\left(\bar{S}_{t_{i}}^{(n)}\right)_{0 \leq i \leq m}, n \geq 0\right\}$ shares an averaging property with respect to a distribution $\nu$ as developed in 22 and is bounded by a real number $L \in(0,+\infty)$ so that $\bar{S}^{(n)} \in[0, L]^{m+1}$ for every $n \geq 1$. Moreover, we will need to prove the existence of a pathwise Lyapunov function, which means in this one dimensional setting that $H\left(\cdot,\left(\left(s_{t_{i}}\right)_{0 \leq i \leq m}\right)\right.$ is non-decreasing for every $\left(s_{t_{i}}\right)_{0 \leq i \leq m} \in \mathbb{R}_{+}^{m+1}, n \geq 1$.

Theorem 2.2. Let $\lambda(x)=A e^{-k x}, A>0, k>0$. Assume $\left(\bar{S}^{(n)}\right)_{n \geq 1}$ is an $[0, L]^{m+1}$-valued $\nu$-averaging sequence where $\nu$ is a probability measure on $\left(\mathbb{R}^{m+1}, \mathcal{B}\right.$ or $\left.\left(\mathbb{R}^{m+1}\right)\right)$. Assume that the execution cost function $C$ is strictly convex over $\left[0, \delta_{\max }\right]$ with $\bar{C}^{\prime}(0)<0$ and $\bar{C}^{\prime}\left(\delta_{\max }\right)>0$. Furthermore, assume that the step sequence $\left(\gamma_{n}\right)_{n \geq 1}$ is a positive non-increasing sequence satisfying

$$
\sum_{n \geq 1} \gamma_{n}=+\infty, \quad n D_{n}^{*}(\bar{S}) \gamma_{n} \underset{n \rightarrow \infty}{\longrightarrow} 0, \quad \text { and } \quad \sum_{n \geq 1} n D_{n}^{*}(\bar{S}) \max \left(\gamma_{n}^{2},\left|\gamma_{n+1}-\gamma_{n}\right|\right)<+\infty,
$$

where $D_{n}^{*}(\bar{S}):=\sup _{x \in[0, L]^{m+1}}\left|\frac{1}{n} \sum_{k=1}^{n} \mathbb{1}_{\llbracket 0, x \rrbracket}\left(\bar{S}^{(k)}\right)-\nu(\llbracket 0, x \rrbracket)\right|$ denotes the discrepancy at the origin of the sequence $\bar{S}$ and satisfies $D_{n}^{*}(\bar{S}) \underset{n \rightarrow \infty}{\longrightarrow} 0$. Finally, assume that

$$
Q_{T} \geq 2 T \lambda\left(-\bar{S}_{0}\right) \quad \text { and } \quad \kappa \leq \frac{1+k\left(\bar{S}_{0}-\delta_{\max }\right)}{k\|\bar{S}\|_{\infty}\left(\Phi\left(Q_{T}\right)-\Phi\left(Q_{T}-1\right)\right)}
$$

Then the recursive procedure defined by (14) converges a.s. towards its target $\delta^{*}=\operatorname{argmin}_{\delta \in\left[0, \delta_{\max }\right]} \bar{C}(\delta)$.

\subsubsection{Criteria for the convexity and monotony at the origin}

In this section, we look for simple criteria involving the parameter $\kappa$, that imply the requested assumption on the execution cost function $C$ (or $\bar{C}$ ) without never needing to really specify the process $S$. The original form of the criteria implying that $C^{\prime}(0)<0$ and $C^{\prime \prime} \geq 0$ cannot really be used in practice since they involve ratios of expectations of functionals combining both the dynamics of the asset $S$ and the execution parameters in a highly nonlinear way. The key establishing the criteria is the functional co-monotony principle established in 21,28 for a wide class of diffusions and their associated time discretization schemes.

A Borel functional $F: \mathbb{D}([0, T], \mathbb{R}) \rightarrow \mathbb{R}$ is non-decreasing (resp. non-increasing) if

$$
\forall \alpha, \beta \in \mathbb{D}([0, T], \mathbb{R}), \quad(\forall t \in[0, T], \alpha(t) \leq \beta(t)) \Longrightarrow F(\alpha) \leq F(\beta) \quad(\text { resp. } F(\alpha) \geq F(\beta)) .
$$

Two functionals $F$ and $G$ on $\mathbb{D}([0, T], \mathbb{R})$ are co-monotonic if they have the same monotony. A functional $F$ has polynomial growth if

$$
\exists r>0 \quad \text { s.t. } \quad \forall \alpha \in \mathbb{D}([0, T], \mathbb{R}), \quad|F(\alpha)| \leq K\left(1+\|\alpha\|_{\infty}^{r}\right) .
$$

Definition 2.3. A càdlàg (resp. continuous) process $\left(S_{t}\right)_{t \in[0, T]}$ satisfies a functional co-monotony principle if for every pair $F, G: \mathbb{D}([0, T], \mathbb{R}) \rightarrow \mathbb{R}$ of co-monotonic Borel functionals (resp. continuous at every $\alpha \in \mathcal{C}([0, T], \mathbb{R})$ for the sup-norm) with polynomial growth such that $F(S), G(S)$ and $F(S) G(S) \in L^{1}$, one has

$$
\mathbb{E}\left[F\left(\left(S_{t}\right)_{t \in[0, T]}\right) G\left(\left(S_{t}\right)_{t \in[0, T]}\right)\right] \geq \mathbb{E}\left[F\left(\left(S_{t}\right)_{t \in[0, T]}\right)\right] \mathbb{E}\left[G\left(\left(S_{t}\right)_{t \in[0, T]}\right)\right] .
$$

Theorem 2.4. Assume that $\left(S_{t}\right)_{t \in[0, T]}$ satisfies a functional co-monotony principle. Assume that $\lambda(x)=A e^{-k x}$, $x \in \mathbb{R}, A, k \in(0,+\infty)$. Then the following monotony and convexity criteria hold true.

(a) Monotony at the origin:

$$
C^{\prime}(0)<0 \quad \text { as soon as } \quad Q_{T} \geq 2 T \lambda\left(-S_{0}\right) \quad \text { and } \quad \kappa \leq \frac{1+k S_{0}}{k \mathbb{E}\left[S_{T}\right]\left(\Phi\left(Q_{T}\right)-\Phi\left(Q_{T}-1\right)\right)} .
$$


(b) ConveXity. Let $\rho_{Q} \in\left(0,1-\left.\frac{\mathbb{P}\left(N^{\mu}=Q_{T}-1\right)}{\mathbb{P}\left(N^{\mu} \leq Q_{T}-1\right)}\right|_{\mu=T \lambda\left(-S_{0}\right)}\right)$. If $\Phi \neq \mathrm{id}$, assume that $\Phi$ satisfies

$$
\forall x \in\left\{1,2, \ldots, Q_{T}-1\right\}, \quad \Phi(x)-\Phi(x-1) \leq \rho_{Q}(\Phi(x+1)-\Phi(x)) .
$$

If $Q_{T} \geq 2 T \lambda\left(-S_{0}\right)$ and $\kappa \leq \frac{2}{k \mathbb{E}\left[S_{T}\right] \Phi_{\ell}^{\prime}\left(Q_{T}\right)}$, then $C^{\prime \prime}(\delta) \geq 0, \delta \in\left[0, \delta_{\max }\right]$, so that $C$ is convex on $\left[0, \delta_{\max }\right]$.

(c) The same inequalities hold for the Euler scheme of $\left(S_{t}\right)_{t \in[0, T]}$ with step $\frac{T}{m}$, for $m \geq m_{b, \sigma}$, or for any $\mathbb{R}^{m+1}$-time discretization sequence $\left(S_{t_{i}}\right)_{0 \leq i \leq m}$ which satisfies a co-monotony principle.

\subsection{Interests rates swap hedging under bid-ask spread costs using stochastic optimization}

We present here a summary of the methodology and results detailed in [30]. We consider a procedure to evaluate and hedge a derivative product in an incomplete market. We are interested in the specific case of hedging a fixed rate against floating interest rate swap in the presence of bid-ask costs. In Section 2.2.1 we present the numerically tractable version of the methodology that we used. Then, in Section 2.2.2, we discuss a more financially sensible methodology and present some of its properties.

\subsubsection{Methodology to optimize the hedging strategy of the swap instrument}

Swap Replication : Product. A swap pays a fixed rate against a floating linear rate. Having a timeline $T_{0}<\cdots<T_{N}$, its payoff is for $1 \leqslant i \leqslant N,\left(T_{i}-T_{i-1}\right)\left(K-L\left(T_{i-1}, T_{i-1}, T_{i}\right)\right)$ payed in $T_{i}$. The fixed rate $K$ can be chosen at the money to make the swap of null value under classical assumption at time $t \leqslant T_{0}$. The linear rate can be computed from the Zero Coupon bond prices : $L\left(T_{F}, T_{B}, T_{E}\right)=\frac{1}{T_{E}-T_{B}}\left(\frac{B\left(T_{F}, T_{B}\right)}{B\left(T_{F}, T_{E}\right)}-1\right)$.

Rate Curve Model. The dynamics of the short rate under the risk neutral probability measure is assumed to be known. It is chosen as an Ornstein Uhlenbeck diffusion. The random variables that define the interest rate market are therefore Gaussian. The Zero Coupon bond prices : $B(t, T)=\mathbb{E}_{t}\left[e^{-\int_{t}^{T} r(s) d s}\right]$ can therefore be computed since $\int_{t}^{T} r(s) d s$ is Gaussian. There exists an optimal discrete but dynamic strategy that hedges perfectly such a swap instrument without liquidity cost. The amounts to be traded are the inverse of the value of the Zero Coupon bond, thus the exponential of a linear combination of Gaussian random variables. The details of the calculation can be found in [30].

Bid-Ask Costs: Incomplete market. We consider that the model gives the mid price of the instruments. This means that our mid prices are martingales, under the risk-neutral probability measure. And therefore the value of a portfolio invested in these assets with bid-ask costs is a super-martingale.

Gaussian projection : Definition of control space. The asset prices depend on Gaussian vectors. The optimal control is therefore a measurable function of these Gaussian variables, which we approximate by a linear combination of Hermit polynomials of the Gaussian variables. Without bid-ask costs, a polynomial of degree 3 or 4 gives sufficient accuracy.

Robbins Monro Algorithm : Stochastic optimization. For our numerical simulations, we consider the problem of minimizing the expectation of the hedging error. The price without bid-ask spread costs will be the initial amount of the self-financing strategy.

Using the square of the hedging error gives convex properties to the function to optimize. We therefore apply the Robbins-Monro algorithm on the coefficients of the polynomial that defines the control.

Considering a function of parameter $\pi$, defined as an expectation, that we have to optimize: $f(\pi)=$ $\mathbb{E}[U(\pi, X)]$. The stochastic gradient algorithm defines the sequence of parameters as : $\pi^{n+1}=\pi^{n}-\gamma_{n} \nabla_{\pi} U\left(\pi^{n}, X_{n+1}\right)$ where $\sum_{n} \gamma_{n}=\infty, \sum_{n} \gamma_{n}^{2}<\infty$. Under good assumptions, [7. shows almost sure convergence in dimension one. We use a stabilized version of the algorithm due to Chen and described in 8 . The initial value $\pi_{0}$ of the parameters defining the control have some importance in the optimization.

Numerical Results. The following graphs illustrate our results. 
The left graph in Figure 1 shows the optimization of the parameters under the stochastic optimization algorithm.

The right graph in Figure 1 shows the expectation of the square of the hedging error as a function of the value of the bid ask costs for four different strategies. Two strategies that can be computed explicitely : the null strategy and the optimal strategy without bid-ask spread costs. The two other strategies are optimized by running the stochastic algorithm starting from the previous ones. There are 5 trading dates in the swap.
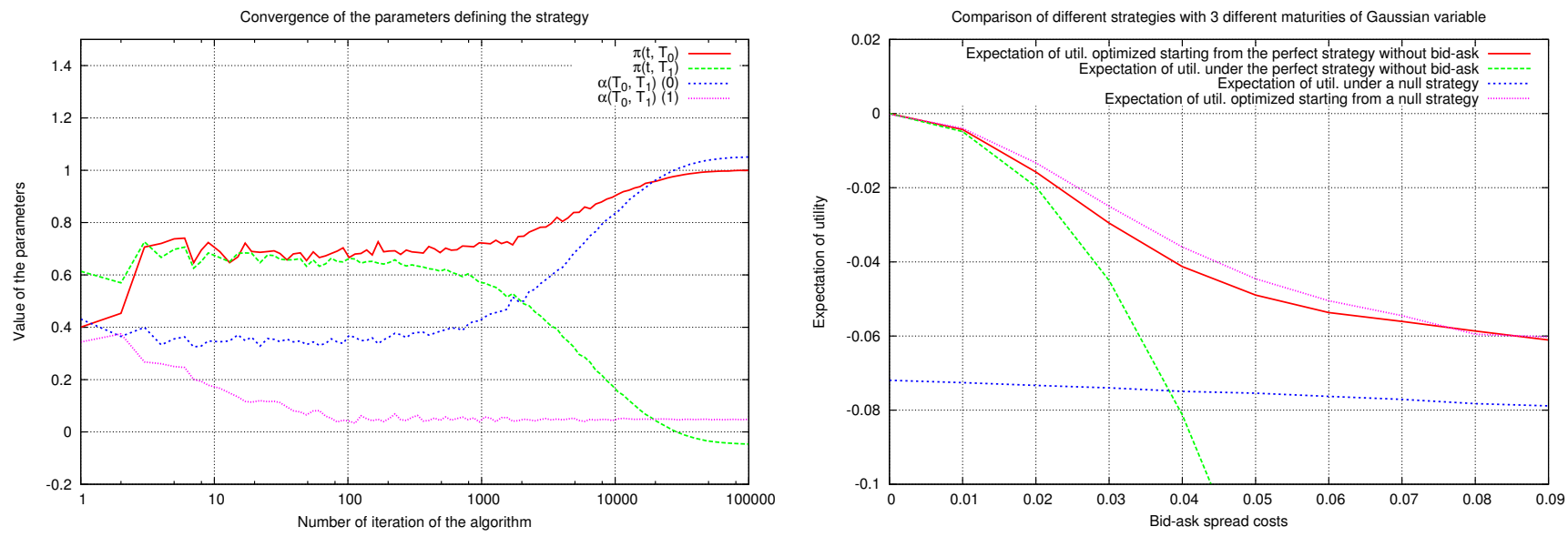

FIGURE 1. Left: parameter optimization with stochastic optimization algorithm. Right: value of the optimized risk measure for different strategies

\subsubsection{Optimization of Risk Measure : Toward Indifference pricing}

Using the square of the hedging error as the criterion to optimize, we observe good convergence properties of the algorithm. But this criterion lacks some good financial properties since it does not discriminate positively the profit from the losses. This choice must only be seen as an approximation for a more meaningful problem : optimizing a risk measure and computing an indifference price. Indeed, replacing the square of the hedging error by an increasing risk measure as defined in [10], deteriorates the convergence properties of the stochastic algorithm. So our approach is similar to the one proposed in [6] where the Value At Risk is used for the optimization and the Conditional Value At Risk for the final check of the efficiency of the strategy.

Let us now explain the methodology to compute indifference prices against a risk measure and propose a general form of the risk measure. We consider having chosen a methodology to transform any portfolio, trading strategy, contract, set of future cash flows into cashes at a time horizon $T$.

Risk Measure. A risk measure $\rho$ is a real function that takes as argument a random variable representing our final wealth. It is concave and increasing. $X \leqslant Y \Rightarrow \rho(X) \leqslant \rho(Y)$ and $\rho(\lambda X+(1-\lambda) Y) \geqslant \lambda \rho(X)+$ $(1-\lambda) \rho(Y)$ where $X$ and $Y$ are two random variable and $\lambda \in[0,1]$.

One of the most general risk measure can be defined as follows : $U$ is a real concave and increasing function, $\phi$ is a real decreasing function, $W$ is our final wealth and $F_{(W)}$ its distribution function : $\int_{0}^{1} \phi(u) U\left(F_{(W)}^{(-1)}(u)\right) d u$.

When $\phi$ is a Heaviside function normalized by the probability of the worst cases and $U$ the identity, one recovers the Conditional Value at risk or Expected Shortfall. When $\phi$ is equal to 1, this is just the expectation of the utility of the final wealth.

Indifference Pricing. We consider a risk measure $\rho$, a set of acceptable strategies $\Pi$. Let $V_{P}^{\pi}(T)$ be the final wealth of a self-financing portfolio under the strategy $\pi$ with initial amount of money $P$. The indifference price 
of a contract with final pay-off $\Psi(T)$ will be the amount of money $P$ such that :

$$
\sup _{\pi \in \Pi} \rho\left(V_{0}^{\pi}(T)\right)=\sup _{\pi \in \Pi} \rho\left(\Psi(T)+V_{P}^{\pi}(T)\right) .
$$

The monotonicity of the risk measure makes this amount unique.

If we add the assumption that the expectation of the cash flow of the contract is finite, we prove that $P$ exists.

We can extend this definition to any set of future cash flows.

No Arbitrage. We assume that the real price has positive bid-ask costs around the mid-price and that the mid-price is a diffusion under a risk neutral probability measure. This market is arbitrage free in the classic way but also when considering indifference pricing arbitrage.

Choice of the probability in the rates framework. In the interest rates market, there is a question of how to choose the probability measure to define the risk measure. Two possibilities seem equally sensible financially : the risk neutral probability measure using the discounted final wealth, or the forward neutral probability measure using the final wealth. The change of probability from risk neutral to forward neutral is the discount factor in the risk neutral framework given by our mid-price. The question is therefore whether this discount factor is inside our utility and distribution function or not. This makes different kind of strategies risk-free wihout bid-ask costs. In the first case it is the strategy of reinvesting instantaneously our wealth at the risk free rate and in the second case it is the strategy of investing all our money at maturity.

\section{REFERENCES}

[1] A. Ahdida and A. Alfonsi. A Mean-Reverting SDE on Correlation matrices. Stochastic Process. Appl., 123(4):1472-1520, 2013.

[2] A. Ahdida and A. Alfonsi. Exact and high order discretization schemes for Wishart processes and their affine extensions. Ann. Appl. Probab., 23(3):1025-1073, 2013.

[3] A. Alfonsi. On the discretization schemes for the CIR (and Bessel squared) processes. Monte Carlo Methods Appl., 11(4):355$384,2005$.

[4] A. Alfonsi. High order discretization schemes for the CIR process: application to affine term structure and Heston models. Math. Comp., 79(269):209-237, 2010.

[5] V. Bally and D. Talay. The law of the Euler scheme for stochastic differential equations. I. Convergence rate of the distribution function. Probab. Theory Related Fields, 104(1):43-60, 1996.

[6] O. Bardou, N. Frikha, and G. Pagès. Computing VaR and CVaR using stochastic approximation and adaptive unconstrained importance sampling. Monte Carlo Methods Appl., 15(3):173-210, 2009.

[7] J. R. Blum. Approximation methods which converge with probability one. Ann. Math. Statistics, 25:382-386, 1954.

[8] H.F. Chen and Y.M. Zhu. Stochastic approximation procedures with randomly varying truncations. Sci. Sinica Ser. A, 29(9):914-926, 1986.

[9] Q. Dai and K. Singleton. Specification analysis of affine term structure models. The Journal of Finance, LV(5):1943 - 1978, 2000.

[10] J.-F. Delmas. Mesures de risque en finance. Cours de la chaire Risques Financiers (Ecole Polytechnique, Ecole des Ponts, Société Générale)., 2009.

[11] T. Fujiwara. Sixth order methods of kusuoka approximation. 2006.

[12] M.B. Giles. Multilevel Monte Carlo path simulation. Oper. Res., 56(3):607-617, 2008.

[13] J. Guyon. Euler scheme and tempered distributions. Stochastic Process. Appl., 116(6):877-904, 2006.

[14] S. Heston. A closed-form solution for options with stochastic volatility with applications to bond and currency options. The Review of Financial Studies, 6(2):327-343, 1993.

[15] J. Jacod and A. N. Shiryaev. Limit theorems for stochastic processes, volume 288 of Grundlehren der Mathematischen Wissenschaften [Fundamental Principles of Mathematical Sciences]. Springer-Verlag, Berlin, second edition, 2003.

[16] I. Karatzas and S. Shreve. Brownian Motion and Stochastic Calculus. Springer-Verlag, 2 edition.

[17] A. Kebaier. Statistical Romberg extrapolation: a new variance reduction method and applications to option pricing. Ann. Appl. Probab., 15(4):2681-2705, 2005.

[18] H. J. Kushner and D. S. Clark. Stochastic approximation methods for constrained and unconstrained systems, volume 26 of Applied Mathematical Sciences. Springer-Verlag, New York, 1978.

[19] H. J. Kushner and G. G. Yin. Stochastic approximation and recursive algorithms and applications, volume 35 of Applications of Mathematics (New York). Springer-Verlag, New York, second edition, 2003. Stochastic Modelling and Applied Probability. 
[20] O.A. Ladyzenskaja, V.A. Solonnikov, and N.N. Ural'tseva. Linear and Quasi-linear Equations of Parabolic Type. Number 23 in Translations of Mathematical Monographs. 1988.

[21] S. Laruelle, C.-A. Lehalle, and G. Pagès. Optimal posting price of limit orders: learning by trading. Math. Financ. Econ., 7(3):359-403, 2013.

[22] S. Laruelle and G. Pagès. Stochastic approximation with averaging innovation applied to finance. Monte Carlo Methods Appl., 18(1):1-51, 2012.

[23] M. Martinez and D. Talay. Discrétisation d'équations différentielles infinidimensionnelles à générateur sous forme divergence avec coefficients discontinu. C.R. Acad. Sci. Paris Ser. I, 342:51-56, 2006.

[24] S. Niklitschek-Soto. PhD thesis, in preparation under the supervision of D. Talay.

[25] M. Ninomiya and S. Ninomiya. A new higher-order weak approximation scheme for stochastic differential equations and the Runge-Kutta method. Finance and Stochastics, 13:415-443, 2009.

[26] S. Ninomiya and N. Victoir. Weak approximation of stochastic differential equations and application to derivative pricing. Appl. Math. Finance, 15(1-2):107-121, 2008.

[27] K. Oshima, J. Teichmann, and D. Velušček. A new extrapolation method for weak approximation schemes with applications. Annals of Applied Probability, 22(3):1008-1045, 2012.

[28] G. Pagès. A functional co-monotony principle with an application to peacoks. 2012. to appear in Séminaire de probabilités.

[29] G. Strang. On the construction and comparison of difference schemes. SIAM J. Numer. Anal., 5:506-517, 1968.

[30] D. Talay, E. Tanré, V. Reutenauer, and C. Michel. Hedging interests rates derivatives in presence of bid-ask spread using stochastic optimization. Submitted, 2012.

[31] D. Talay and L. Tubaro. Expansion of the global error for numerical schemes solving stochastic differential equations. Stochastic Anal. Appl., 8(4):483-509 (1991), 1990. 